\title{
La actividad ecoturística y su incidencia en la conservación ambiental del Jardín Botánico Las Orquídeas del sector Los Ángeles de Puyo, Pastaza, Ecuador
}

\author{
Ecotourism activity and its impact on the \\ environmental conservation of "The Orchids" \\ Botanical Garden in the Los Ângeles de Puyo \\ sector, Pastaza, Ecuador
}

Víctor Hugo del Corral', Darwin Sucoshañay², Luis Álvarez ${ }^{3}$, Patricio Castro ${ }^{4}$

Resumen. Ecuador se encuentra entre los 17 países de mayor biodiversidad en el mundo por sus características topográficas, su diversidad de zonas climáticas y una enorme población de especies de flora y fauna. El objetivo general de este trabajo fue estudiar la incidencia de la actividad ecoturística en la conservación ambiental del atractivo turístico Jardín Botánico Las Orquídeas (El Jardín) del sector Los Ángeles de Puyo, Pastaza, Ecuador. Se utilizaron instrumentos de recolección de información como la encuesta a moradores del sector y turistas. Las modalidades especiales de la investigación consistieron en elaborary desarrollar un modelo operativo variable, para solucionar problemas de grupos sociales de carácter socioeconómico - productivo con la ayuda de implementación de propuestas de desarrollo. Los resultados permitieron confirmar que la conservación ambiental incide en la actividad ecoturística. Se determinó que El Jardín posee un potencial recurso de conservación de la flora, razón por la cual tiene la categoría de conservación de la naturaleza, teniendo como fortaleza la gran variedad de orquídeas recolectadas y la recuperación de la selva. Sin embargo, la sobrepoblación de sus alrededores va haciendo difícil el mantener un ecoturismo sostenible.

Palabras clave: Atractivo turístico, conservación ambiental, ecoturismo sustentable, ecoturismo comunitario.
Abstract. Ecuador is among the 17 countries with the greatest biodiversity in the world due to its topographic features, diversity of climatic zones and an enormous population of flora and fauna species. The general aim of this work was to study the impact of ecotourism activity on the environmental conservation tourist attraction "The Orchids" Botanical Garden (The Garden) in the Los Ángeles de Puyo sector, Pastaza, Ecuador. Data collection instruments such as the survey administered to sector inhabitants and tourists were used. The special modalities of the study consisted of preparing and developing a variable operative model to solve social group issues of a socioeconomic-productive nature with the aid of the implementation of development proposals. The results confirmed that environmental conservation affects ecotourism activity. It was determined that The Garden has a potential conservation resource for flora; therefore, it has nature conservation status, with its strength being the great variety of harvested orchids and the recovery of the forest. However, the overcrowding of its surroundings is making it difficult to maintain sustainable ecotourism.

Key words: Tourist attraction, environmental conservation, sustainable ecotourism, community-based ecotourism.

\section{(Presentado: 22 de marzo de 2017. Aceptado: 26 de mayo de 2017)}

1 Doctor (c) en Ciencias Económicas con especialización en turismo. Universidad Estatal Amazónica, Ecuador. E-mail: vdelcorralquea.edu.ec 2 Doctor en Ciencias Geográficas, Secretaría Nacional de Educación Superior, Ciencia, Tecnología e Innovación, Ecuador. E-mail: javier.dsv6263वgmail.com 3 Master Ejecutivo en Dirección de Empresas. Doctor (c) en Economía Turística, Universidad de la Habana, Cuba. E-mail: lalvarezQuea.edu.ec 4 Doctor (c). Consultor Senior en Turismo, Medio Ambiente y TIC's de PROYECTUR. E-mail: patricastro ayahoo.com 


\section{INTRODUCCIÓN}

Ecuador constituye un escenario ideal para el ecoturismo y un espacio de múltiples atractivos culturales y naturales. Ecuador se encuentra entre los 17 países de mayor biodiversidad en el mundo por sus características topográficas, su diversidad de zonas climáticas y una enorme población de especies de flora y fauna. Esta abundancia biológica se refleja en una amplia variedad de seres vivientes. De acuerdo al Ministerio de Turismo el $10 \%$ de todas las especies de plantas vasculares y $18 \%$ de todas las especies de aves existentes en el mundo se encuentran en el Ecuador, cifra que representa el $0,2 \%$ de total de la superficie terrestre. En Ecuador la biodiversidad se ve favorecida por la presencia de cuatro regiones naturales: la Costa del Pacífico, los Andes, la Amazonía y el Archipiélago de Galápagos; específicamente la Amazonía es hábitat de una gran diversidad de especies de flora y fauna. También son importantes las áreas protegidas existentes en el Ecuador y un escenario cubierto de ríos, lagos, lagunas, pantanos y montañas. En esta región diversas comunidades han comenzado a incursionar en el ecoturismo como una fuente de recursos y una herramienta para mejorar sus condiciones de vida. La Amazonía se encuentra en la parte oriental del país donde las temperaturas son altas, la pluviosidad es constante y la humedad elevada. Su vegetación es exuberante, así como su fauna. Inmensos territorios protegidos y varias nacionalidades indígenas conviven con tradiciones y costumbres propias y distintas. Seis provincias ecuatorianas conforman la Amazonía.

Toda esta riqueza forestal, que ha permitido explotar como atractivos turísticos para el desarrollo ecoturístico, se ve ampliamente amenazada por la pérdida de los bosques o la deforestación, siendo ellos algunos de los problemas más graves del país y del mundo. Como consecuencia de esto se han observado impactos relevantes, tales como un aumento de la destrucción del suelo debido a la erosión, pérdida del hábitat de la vida silvestre, pérdida de biodiversidad y alteración del ciclo del agua. La deforestación ocasiona también una pérdida inmensa de dinero, porque la mayoría de los árboles derribados no son utilizados, sino quemados y muchas veces ni siquiera se los utiliza para carbón o leña.

Pastaza es la provincia más grande del Ecuador, con una superficie de $29.000 \mathrm{~km} 2$, pero también tiene la población más baja de todas las provincias del Ecuador, con 61.400 habitantes. Por eso la densidad poblacional es comparativamente baja, de sólo 4,7 hab/km2. Según datos del Instituto para el Ecodesarrollo Regional Amazónico (ECORAE), la mayoría de la gente vive en el oeste de la provincia en los centros urbanos, mientras que el este es muy poco poblado, más que todo de grupos indígenas. La cobertura del suelo todavía es $91 \%$ de bosque primario, el $4 \%$ bosque secundario y $4 \%$ de agricultura, principalmente plátano, caña, naranjilla, yuca y ganado. El $56 \%$ de la población vive en el área rural y el $40 \%$ de la población total vive de la agricultura, silvicultura o caza (ECORAE 2003). El petróleo todavía no está siendo extraído a gran escala y los suelos pobres de la Amazonía no permiten los cultivos agrícolas a largo plazo.

En vista de que la cobertura forestal posee el 95\% de la superficie total, la extracción, comercialización, preparación y manufactura de madera tienen un valor importante en Pastaza. Por esto la madera es la materia prima más importante en la provincia, lo cual ha llevado a que su explotación afecte directamente a la gran biodiversidad que tiene la selva amazónica de Pastaza, que sufre la intervención del hombre a través de la tala indiscriminada de árboles, introduce la ganadería con pastos de mala calidad y desarrolla una actividad agrícola muy rudimentaria. Todo esto ha traído como consecuencia la pérdida de especies de árboles autóctonos de la región y la afectación de los suelos por un mal manejo agronómico. El ritmo de explotación de la selva se efectúa arbitrariamente sin técnicas forestales apropiadas, trayendo como consecuencia la pérdida de especies forestales de gran valor; no existen viveros forestales para la repoblación forestal, la ganadería es muy rudimentaria y afecta la calidad de los suelos. Los cultivos agrícolas tienen muy bajos rendimientos debido a que los suelos poseen características muy ácidas, baja fertilidad, gran contenido de metales pesados y un alto porcentaje de materia orgánica de mala calidad, propiciándose procesos erosivos en la región.

El sector de los Ángeles, aledaño a la ciudad de Puyo, capital de la provincia amazónica Pastaza, es un sitio natural e intervenido por el hombre, cambiando así su paisaje geográfico. Esto ha permitido que hombres vi- 
sionarios rescaten un área de terreno para convertirlo en uno de los atractivos más importantes de esta ciudad, como es El Jardín Botánico Las Orquídeas. Este es un lugar único, que resulta ser la muestra patente de que restaurar el bosque tropical luego de haber sido explotado y destruido es posible. Este jardín reúne en sus instalaciones orquídeas y árboles representativos de la región, ordenados en secciones de acuerdo a su aplicación y utilidad.

A pesar de esta gran riqueza que ofrece el atractivo turístico, para los miembros de la comunidad y aledaños las condiciones de vida son muy deficientes. Es que la presencia de los visitantes nacionales y extranjeros a este atractivo no es aprovechada por sus moradores para establecer otras actividades que permitan que lo disfruten, sean éstos en los aspectos de gastronomía, artesanía y manifestaciones culturales, debido a que su población mayormente pertenece a la etnia quichua. Se puede apreciar que la riqueza cultural de idioma, su artesanía, su arquitectura y modo de vida se sigue manteniendo y cultivando fuera de su contexto, debido a la acción de aculturización del que han sido objeto las culturas indígenas, y en lugar de ser un orgullo, se va perdiendo tanta riqueza y adaptando costumbres ajenas a las suyas, perdiendo así su identidad. A esto se suma la pérdida de vivir en colectividad, en comunidad, cambiando a una vida unilateral, donde los hombres por buscar beneficios salen a buscar trabajo en otros lugares, principalmente en las compañías petroleras, perdiendo el sentido de desarrollo comunitario. Desde esta perspectiva el atractivo turístico se ve amenazado por la afluencia de moradores que destruyen su entorno para transformar áreas de construcción de viviendas, sin considerar que pueden aprovechar este espacio para su mejoramiento económico. La destrucción de la selva tropical de su entorno hace que al hábitat de la flora existente en el atractivo se le dedique más cuidado, trayendo como consecuencia mayor egreso económico y menor afluencia de visitantes. Este escenario podría cambiar si se logra desarrollar una investigación que presente a su misma comunidad la riqueza natural y cultural como una alternativa de desarrollo económico comunitaria, con la participación de ellos como aporte a su desarrollo ecoturístico.

\section{MARCO CONCEPTUAL}

\section{Ecoturismo}

Es un enfoque para las actividades ecoturísticas en el cual se privilegia la sustentabilidad, la preservación y la apreciación del medio, tanto natural como cultural, que acoge a los viajantes. El turismo de la naturaleza ofrece muchas opciones, ya que desarrolla actividades vinculadas para proteger las riquezas naturales existentes, para poner en función social diferentes escenarios naturales (Peralta, 2004). Aunque existen diferentes interpretaciones, por lo general el turismo ecológico se promueve como un turismo "ético", en el cual también se presume como primordial el bienestar de las poblaciones locales, y tal presunción se refleja en la estructura y funcionamiento de las empresas, grupos y cooperativas que se dedican a ofrecer tal servicio.

\section{Turismo}

El turismo como tal nace en siglo XIX, como una consecuencia de la Revolución Industrial, con desplazamientos cuya intención principal es el ocio, descanso, cultura, salud, negocios, relaciones familiares y recreación en lugares no habituales. Estos movimientos se caracterizan por su finalidad de otros tipos de viajes motivados por guerras, movimientos migratorios, conquista y comercio, entre otros. No obstante, el turismo tiene antecedentes históricos claros de movilidad con un objetivo de descanso y conocer lugares poco frecuentados en sedentarismo.

Es posible afirmar que los viajes de placer tuvieron sus inicios en los últimos años del siglo XIX y principios del XX; grandes cambios en la sociedad, en los estilos de vida en la industria y la tecnología, alteraban la morfología de la comunidad; hay en la historia momentos de cambios excepcionales y de enorme expansión; el siglo XIX fue testigo de una gran expansión económica, seguida de una revolución industrial y científica, incluso mayor en el siglo XX; el turismo fue uno de los pilares beneficiarios para llegar a ser a finales del siglo XX la mayor industria del mundo que a la par del ambiente desarrollan y diversifican las economías mundiales. 
Según datos del "Plan de Desarrollo Turístico Local 2005 2009" del Municipio de Pastaza, entre 1950 y 1973 se comienza a hablar del boom turístico. Se considera al turismo como el "conjunto de las acciones que una persona lleva a cabo mientras viaja y pernocta en un sitio diferente al de su residencia habitual, por un periodo consecutivo que resulta inferior a un año". "En la actualidad el concepto de turismo planteado desde los sectores económicos, por el capital, ha perdido el romanticismo inicial, y este fenómeno socioeconómico ha sido estudiado, legislado y explotado, por lo que en la actualidad se establecen distintas clases de turismo, destinados a uno u otro público, en referencia a su poder adquisitivo, a su poder económico, a sus gustos, a sus preferencias, a sus inquietudes... etc.”.

\section{Turismo científico}

El turismo científico es una modalidad de turismo cuya motivación es el interés en la ciencia o la necesidad de realizar estudios e investigaciones científicas en lugares especiales, como estaciones biológicas o yacimientos arqueológicos. En ocasiones existe la necesidad de viajar para observar in situ alguna realidad que es objeto de estudio. El turismo científico se realiza de forma individual o en pequeños grupos para evitar alterar el objeto de estudio en un entorno natural.

\section{Desarrollo sostenible}

Se llama desarrollo sostenible a aquel desarrollo que es capaz de satisfacer las necesidades actuales sin comprometer los recursos y posibilidades de las futuras generaciones. Intuitivamente una actividad sostenible es aquella que se puede mantener; por ejemplo, cortar árboles de un bosque asegurando la repoblación es una actividad sostenible. Por el contrario, consumir petróleo no es sostenible con los conocimientos actuales, ya que no se conoce ningún sistema para crear petróleo a partir de la biomasa. Hoy sabemos que una buena parte de las actividades humanas no son sostenibles a mediano y largo plazo, tal y como hoy están planteadas.

\section{Jardines botánicos}

Los jardines botánicos (del latín Hortus botanicus) son instituciones habilitadas por un organismo público, privado o asociativo (en ocasiones la gestión es mixta), cuyo objetivo es el estudio, la conservación y divulgación de la diversidad vegetal. Se caracterizan por exhibir colecciones científicas de plantas vivas, que se cultivan para conseguir alguno de estos objetivos: su conservación, investigación, divulgación y enseñanza. En los jardines botánicos se exponen plantas originarias de todo el mundo, generalmente con el objetivo de fomentar el interés de los visitantes hacia el mundo vegetal, aunque algunos de estos jardines se dedican, exclusivamente a determinadas plantas y a especies concretas.

\section{Conservación ambiental}

Proviene del latín conservatio, la conservación es la acción y efecto de conservar (mantener, cuidar o guardar algo, continuar una práctica de costumbres). El término tiene aplicaciones en el ámbito de la naturaleza, la alimentación y la biología, entre otros. "La conservación ambiental es el uso racional y sostenible de los recursos naturales y el ambiente. Entre sus objetivos está garantizar la persistencia de las especies y los ecosistemas y mejora de la calidad de vida de las poblaciones, para el beneficio de la presente y futuras generaciones". Se considera importante enunciar que en la actualidad se entiende por "conservación ambiental, conservación de las especies, conservación de la naturaleza o protección de la naturaleza como las distintas formas de proteger y preservar para el futuro la naturaleza, el medio ambiente o específicamente alguna de sus partes: la flora y la fauna, las distintas especies, los distintos ecosistemas, los valores paisajísticos, etc.".

La conservación ambiental o conservación de las especies, por ejemplo, hace referencia a la protección de los animales, las plantas y el planeta en general. Esta conservación apunta a garantizar la subsistencia de los seres humanos, la fauna y la flora, evitando la contaminación y la depredación de recursos.

Esta posición en la práctica diaria se ha visto privado principalmente por el consumo excesivo del ser humano, por la contaminación, por el descuido de nuestros ríos, especies y toda la naturaleza. Entre los principales problemas ambientales se podrían enunciar la deforestación, contaminación y basura. Ante esta realidad se debe propender a realizar un cambio social dirigido a promover la mejora de la calidad de vida de las sociedades humanas, en el cual el aprovechamiento de los recursos naturales y el ambiente se realice en forma armónica, garantizándose su utilización por parte de la presente y futuras generaciones. 
La conservación de la diversidad biológica debe ser asumida desde cuatro puntos de vista: ecológico, ético o cultural, económico y social.

- $\quad$ Ecológico: Se refiere a la protección de cuencas hidrográficas y de áreas sensibles a la erosión y el control de la sedimentación. A la protección y mantenimiento de programas de protección de las especies en peligro de extinción y de limpieza de elementos químicos nocivos, especialmente en la industria. Renovar determinados recursos vitales que han sido considerados como inagotables, pero que hoy tienden a ser escasos, tales como los suelos, el agua y el aire.

- $\quad$ Ético o cultural: Se refiere al derecho que cada ser vivo tiene para existir, sin importar la utilidad o no para el ser humano. Se basa en el respeto hacia la naturaleza. La diversidad biológica no se limita al mundo de las plantas y los animales, incluye también la diversidad cultural humana. Esta se expresa en la multiplicidad de lenguas y religiones, mitos, símbolos, creencias, expresiones artísticas, estructuras sociales y formas de manejo de los recursos de las comunidades locales y poblaciones indígenas. La pérdida de diversidad cultural podría tener un efecto tan devastador como la extinción de las especies. Cuando se deslaza a estos pueblos o culturas, o se ven amenazadas por el progreso, también queda destruida su gran riqueza de conocimientos; y un recurso valioso para la comunidad global se pierde para siempre.

- $\quad$ Económico: Se refiere al aporte de los recursos, las especies y la información genética para el desarrollo. La biodiversidad constituye un patrimonio natural para las generaciones actuales y futuras. La agricultura, la pesca, la industria maderera, la acuicultura y el ecoturismo se basan, en gran medida, en los recursos que ofrece la naturaleza. Muchos recursos naturales y servicios ambientales no pueden ser valorados por las reglas del mercado.

Hoy la tendencia es buscar la compatibilidad entre la defensa del medio ambiente y el desarrollo económico social, como base de una nueva visión económica: el desarrollo sostenible, que es un modelo de desarrollo centrado en la búsqueda del mejoramiento de la calidad de la vida humana, sin rebasar la capacidad de carga de los ecosis- temas de la Tierra, de manera que los beneficios de la naturaleza y la sociedad alcancen no sólo para las presentes generaciones sino para las venideras.

\section{Recursos naturales}

Se denominan recursos naturales a aquellos bienes materiales y servicios que proporciona la naturaleza sin variación por parte del ser humano, y que son valiosos para las sociedades humanas por ayudar a su bienestar y desarrollo de manera directa. Se define que "los recursos naturales son aquellos bienes que pueden obtenerse de la naturaleza sin mediar la intervención de la mano del hombre. Los recursos naturales tienen una influencia positiva en la economía al ayudar a su desarrollo y satisfacer necesidades de la población".

Los recursos naturales son los materiales de la naturaleza que los seres humanos pueden aprovechar para satisfacer sus necesidades de alimento, vestido, vivienda, educación, cultura y recreación, entre otros. Los recursos naturales son la fuente de las materias primas (madera, minerales, petróleo, gas y carbón), que transformados sirven para producir bienes muy diversos.

\section{La selva}

La selva es un bioma, es decir un ambiente natural o un paisaje bioclimático, por ende en las zonas selváticas, hay un clima, una fauna y una vegetación característicos. Un ejemplo de bioma es la selva del Amazonas (selva tropical), en América del Sur, es la más extensa del mundo $\left(6.000 .000 \mathrm{~km}^{2}\right)$ y se halla rodeada por el río Amazonas, siendo parte de Brasil y Perú principalmente, y en menor proporción de Colombia, Guyana, Venezuela, Bolivia, la Guayana Francesa y Ecuador.

\section{La biodiversidad}

"La biodiversidad comprende la variabilidad de organismos vivos de cualquier fuente, incluidos, entre otras cosas, los ecosistemas terrestres y marinos, junto con otros ecosistemas acuáticos y los complejos ecológicos de los que forman parte; comprende la diversidad de cada especie, entre las especies y los ecosistemas". De acuerdo a esta conceptualización se suele considerar la biodiversidad formada por tres "componentes" claramente relacionados (Tabla 1). 
TABLA 1. Componentes de la biodiversidad.

Diversidad genética Resume la idea de variedad y variabilidad de genes y agrupaciones de genes. Suele advertirse mediante la identificación de variedades genéticas por debajo de la categoría de especie, pero incluye también la variedad genética individual.
Genes, grado de heterocigosis, variedad, raza, subespecie.

Diversidad taxonómica Es la idea más difundida de biodiversidad, al incluir la variedad de especies; pero también incluye la variedad a otras escalas taxonómicas: géneros, órdenes, clases, reinos.

Especies, géneros, órdenes, clases, troncos, divisiones, phyla, reinos.

\section{Diversidad de ecosistemas}

Supone la variedad de sistemas que incluyen vida.

Ecosistemas, ecoprovincias, Al no existir una clasificación universalmente aceptada de estos sistemas, resulta difícil de estimar

Fuente: José Antonio Pascual Trillo. Elaboración propia (ver http://platea.pntic.mec.es/ jpascual/vida/diver3.htm)

\section{METODOLOGÍA}

\section{Población y muestra}

Toda población necesita ser limitada en forma precisa, es decir, en grupos que coadyuven a la observación de elementos y fenómenos a ser investigados, se debe por lo tanto determinar si cabe investigar a todos o solo a una parte de los involucrados. Para esta investigación se consideraron tres grupos de población: (i) Turistas o visitantes que acceden mensualmente al Jardín Botánico, 100 personas en promedio; (ii) Pobladores locales de la comunidad con sus jefes de hogar, 260 personas; y (iii) Finalmente 1 gerente administrativo del lugar.

\section{Muestra}

Para el cálculo de la muestra se aplicó la siguiente fórmula:

$$
n=\frac{N \times P \times Q}{(N-1)\left(\frac{e}{Z}\right)^{2}+P \times Q}
$$

Donde $\mathrm{n}$ es el tamaño de la muestra, $Z$ el nivel de confianza $(Z=1,96), P$ la probabilidad de ocurrencia $(0,5), Q$ la probabilidad de no ocurrencia $(1-0,5=0,5), \mathrm{N}$ la población $(\mathrm{N}=361)$ y e es el error de muestreo 0,05 (5\%). Reemplazando se obtuvo $\mathrm{n}=128$.

Tipo de investigación

La investigación se basa en un enfoque predominantemente cuali-cuantitativo, por cuanto busca la comprensión de los fenómenos sociales de los habitantes que se encuentran inmersos en la parte periférica del atractivo turístico, asumiendo una realidad dinámica de compromiso y participación. Además de tener un enfoque cualitativo, se considera la parte cuantitativa, por cuanto se apoya en la estadística para poder realizar la cuantificación del desarrollo sostenible de los habitantes del sector, lo cual permite buscar las causas y explicar los hechos que se estudian.

La modalidad de campo, considerando que el estudio sistemático se realizará en el lugar que se producen los hechos relacionados a los aspectos que dificultan las actividades normales en el atractivo turístico, objeto de investigación; la realidad socioeconómica de la población que se asienta alrededor de este atractivo. Para la información científica se sustenta en la bibliografía documental, la misma que permite detectar, ampliar y profundizar diferentes enfoques, teorías, conceptualizaciones y criterios 
de diversos actores sobre una cuestión determinada, basándose en documentos como libros, revistas, periódicos y otras publicaciones. La investigación de tipo bibliográfica-documental permitió elaborar el marco teórico conceptual, sobre la problemática de la investigación; con la finalidad de conocer el criterio de varios autores y corriente con relación a la conservación ambiental, el desarrollo ecoturístico, qué son los jardines botánicos y el turismo, así como una explicación de lo que se entiende por desarrollo socioeconómico de los habitantes. Las modalidades especiales de la investigación consistieron en elaborar y desarrollar un modelo operativo variable, para solucionar problemas de grupos sociales de carácter socioeconómico - productivo con la ayuda de implementación de propuestas de desarrollo. En la Figura 1 se presenta el mapa de ubicación del cantón Pastaza, lugar de localización de este trabajo.

\section{FIGURA 1. Mapa de la ubicación del cantón Pastaza.}

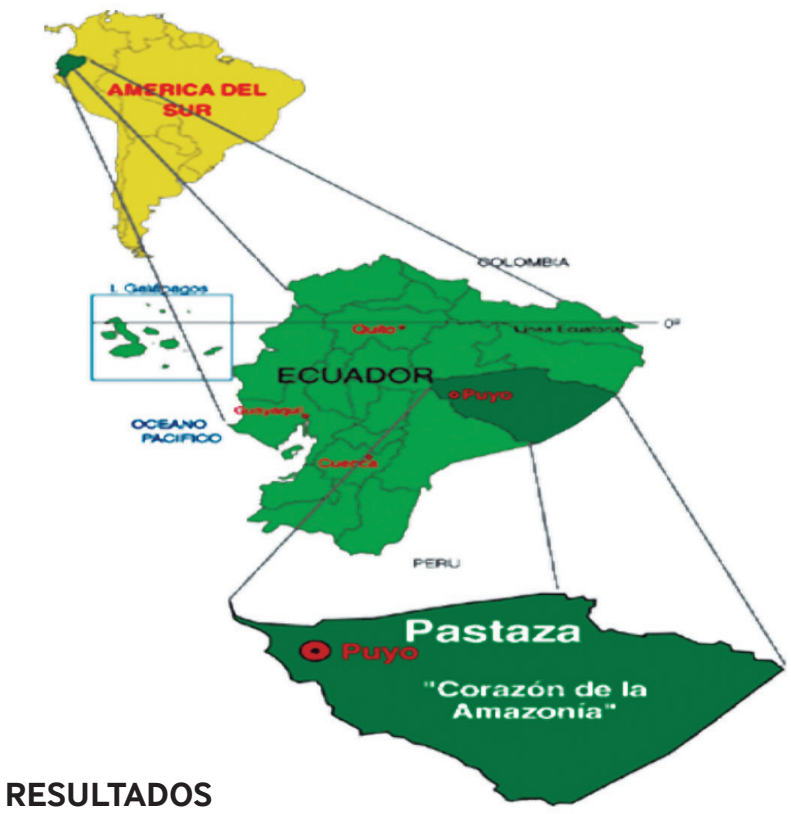

\section{Pregunta 1}

¿Cree usted necesario que se desarrolle la actividad ecoturística en el Jardín Botánico las Orquídeas del sector "Los Ángeles"? La mayoría de los encuestados respondió que sí (91 personas, $71,1 \%$ ). Por lo tanto, el $28,9 \%$ no tiene interés en la actividad ecoturística. Se puede concluir que el grado de interés está en la mayoría de los encuestados, donde se encuentran involucrados los habitantes del sector. Se tiene como base los criterios para mejorar la actividad económica y mejorar el estilo de vida, pues los beneficios serian variados por las visitas de los turistas al sector.

\section{Pregunta 2}

¿Cómo considera que es la participación de la comunidad en la prestación de servicios contiguos al atractivo? La mayoría de los encuestados respondió que es mala (92 personas, $71,9 \%$ ). La mayoría de los encuestados, representado por el $71,9 \%$ manifiestan que es mala; mientras que la minoría, el $28,3 \%$, consideran que tienen una participación muy buena y mencionan que es participativa. Los datos proporcionados reflejan que la población no tiene relación directa con el atractivo; es decir, no existe participación en sus actividades turísticas, lo que muestra que no existe un compromiso de colaborar en su mantención y el cuidado de su medio ambiente, por consiguiente, sus actividades son ajenas a lo que propende el atractivo.

\section{Pregunta 3}

¿La comunidad está integrada en el mantenimiento y servicios que presta este atractivo? La mayoría de los encuestados respondió que no (79 personas, 61,7\%). Los encuestados consideran en un $61,7 \%$ que no se integra a la comunidad en el mantenimiento de los servicios, mientras que un $38,3 \%$ considera que sí lo hacen y sí forman parte del mantenimiento. Los resultados obtenidos se interpretan como una debilidad de la comunidad y el atractivo para trabajar en equipo, así de esta manera sacar adelante el atractivo, conservándolo, cuidándolo y aprovechándolo sostenida y sustentablemente.

\section{Pregunta 4}

¿Considera usted que la actividad ecoturística realizada en el lugar amenaza la conservación y protección del mismo? La mayoría de los encuestados respondió que no (98 personas, 76,6\%). Los habitantes del sector Los Ángeles, en un $76,6 \%$ consideran que el atractivo Las Orquídeas si se ve amenazado en su conservación, mientras que la minoría, representada por el $23,4 \%$ opina que no se ve amenazado. Estos resultados son interpretados como la determinación de que el atractivo Las Orquídeas se vea severamente amenazado en la conservación ambiental, perjudicando su atractivo ecoturístico, 


\section{Pregunta 5}

¿Cree usted que la visita al atractivo satisface la necesidad de los visitantes para experimentar un turismo sustentable y sostenible? La mayoría de los encuestados respondió que no (79 personas, 61,7\%). Los encuestados en su mayoría manifiestan sus expectativas al observar el cuidado ambiental en su parte interna y externa, opinan que no es satisfactorio en un $64,7 \%$, y la minoría que es el $38,3 \%$ opina que sí es satisfactorio el lugar para la visita y aprendizaje de un entorno natural. Los datos expuestos revelan que la apreciación es diversa, consecuencia de los diferentes tipos de turistas, siendo notorio que la conservación ambiental es apreciado y considerado medianamente satisfactorio, criterio relevante que es necesario considerarlo para la vivencia de este atractivo.

\section{Pregunta 6}

¿Si se utiliza ciertos recursos naturales vivientes del lugar, considera necesario renovarlos para minimizar la pérdida de la biodiversidad biológica? La mayoría de los encuestados respondió que sí (75 personas, 58,6\%). Los encuestados del sector Los Ángeles, en una gran mayoría representada por el $58,6 \%$ aceptan que, si se debe minimizar la explotación de recursos naturales, con una renovación de los mismos; mientras que la menor parte que es el $41,4 \%$ no lo aceptan. La comunidad en su mayor porcentaje se encuentra conforme con la minimización de la explotación de recursos naturales, por consiguiente, se observa que los recursos naturales están siendo conservados.

\section{Pregunta 7}

¿Se aplica un ecoturismo responsable, en el atractivo Jardín Botánico las Orquídeas del sector Los Ángeles? La mayoría de los encuestados respondió que sí (73 personas, 57\%). La población de los Ángeles, según sus respuestas el mayor porcentaje que significa el $57 \%$ manifiestan que en el atractivo turístico si se aplica un ecoturismo responsable por parte de los dueños y empleados del mismo; por su parte la minoría representada por un $44 \%$ considera que no. Esto significa que las apreciaciones tanto positivas y negativas se encuentran ligeramente estrechas debido a la ubicación de las viviendas al atractivo turístico lo que genera una opinión segmentada de lo que significa un ecoturismo responsable.

\section{Pregunta 8}

¿Los visitantes al lugar tienen costumbres de conservación y protección a la naturaleza? La mayoría de los encuestados respondió que sí (97 personas, 75,8\%). De conformidad a las respuestas dadas por los encuestados, la mayoría en un $75,8 \%$ consideran que tienen costumbres de conservación, mientras que la menor parte representada por el $24,2 \%$ considera que tienen muy pocas o nulas costumbres de conservación, además opinan que el visitante al lugar no tiene costumbres de protección y conservación. Según los datos obtenidos se aprecia que el atractivo es visitado por diferentes tipos de turistas, lo cual lo hace muy llamativo, considerando que su visita lo hace desde diferentes perspectivas o motivos. Lo importante que cada uno de ellos tendrán diferentes opiniones de su conservación ambiental y aprenderán poco a poco a cuidar de los recursos naturales.

\section{Pregunta 9}

¿El jardín Botánico presenta una imagen limpia, conservada y protegida? La mayoría de los encuestados respondió que sí (79 personas, 61,7\%). Los encuestadores en su mayoría consideran que el atractivo en un $61,7 \%$ presenta una imagen limpia y conservada, mientras que la minoría del 38,3\% manifiestan que no y opina que se observa el lugar poco conservado. Se evidencia que los turistas tienen una buena apreciación a que el atractivo se encuentra en un estado muy limpio y conservado, carta de presentación para que se motive a ser visitado; sin embargo, hay que considerar también el criterio de otros turistas en que hay que mejorar el aseo y conservación y protección del lugar.

\section{Pregunta 10}

¿Se identifica el uso de recursos renovables por parte de los moradores y administración del jardín Botánico las Orquídeas? La mayoría de los encuestados respondió que sí (78 personas, 60,9\%). Sobre el uso de los recursos renovables en el sector de los Ángeles, la mayoría de los encuestados que es el $60,9 \%$ si identifica el uso de recursos renovables, mientras que la minoría del $39,1 \%$ no identifica el uso de estos recursos. Estos resultados determinan que en su mayor porcentaje los habitantes del sector son identificados con el uso de los recursos renovables para la prestación de servicios turísticos.

\section{CONCLUSIONES}

En cuanto a la necesidad de desarrollar la actividad ecoturística en el Jardín Botánico Las Orquídeas la mayoría de los encuestados opinaron que se debe desarrollar esta actividad para beneficio del atractivo, así como de la zona donde ellos habitan; sin embargo, una minoría es indiferente a la actividad ya que consideran que la existencia del 
atractivo no beneficia en gran parte al sector, por lo tanto, la actividad ecoturística que se desarrolle en el mismo no es de su interés.

En base a los resultados de la investigación realizada, acerca de la participación de la comunidad en la prestación de servicios contiguos del atractivo, la mayoría es indiferente a formar parte de la prestación de servicios de dicho atractivo, puesto que carecen de conocimiento respecto a estrategias y beneficios del turismo o ecoturismo, en la cotidianidad de los mismos; por su parte un menor grupo participa en la prestación de servicios turísticos.

Por otra parte, la actividad comercial que los habitantes realizan para obtener ingresos económicos de los turistas que visitan el atractivo turístico Jardín Botánico Las Orquídeas es inadecuada, por lo que no ha permitido tener una actividad económica o comercial normal, lo que ha ocasionado una pérdida de productividad del recurso existente por lo que ésta es poca aprovechada en la comunidad. La integración de la comunidad en el mantenimiento, conservación y servicios que presta el atractivo es muy bajo, ya que se han hecho esfuerzos por parte del atractivo para que la comunidad se una y apoye esta propuesta de actividad turística que a la larga será muy beneficioso para la misma, sin tener mayores resultados.

Finalmente, se determinó que el Jardín Botánico Las Orquídeas posee un potencial recurso de conservación de la flora, razón por la cual tiene la categoría de conservación de la naturaleza, teniendo como fortaleza la gran variedad de orquídeas recolectadas y la recuperación de la selva. Sin embargo, la sobrepoblación de sus alrededores va haciendo difícil el mantener un ecoturismo sostenible.

\section{REFERENCIAS}

Barbault, R., \& Sastrapradja, S.D. (1995). Generation, Maintenance and Loss of Biodiversity. Global BiodiversityAssessment. Cambridge University Press, Cambridge.

Boullon, R. (1992). Las Actividades Turísticas y Recreacionales. Editorial Trillas, México.

Boullon, R. (1997). Planificación del Espacio Turístico. Editorial Trillas, México

Fundación Peralta, José. (1999- 2000). Ecuador - Su Realidad. Séptima Edición. Quito, Ecuador.

Hernández, D. (1996). Proyectos Turísticos Formulación y Evaluación. Editorial Trillas, México.
Hernández, D. (1996). Planificación Turística, un Enfoque Metodológico. Editorial Trillas, México.

Herrera, L., Medina, A., \& Naranjo, G. (2004). Tutoría de la Investigación Científica. Guía para elaborar en forma amena el trabajo de Graduación. Gráficas Corona, Quito, Ecuador.

Hill, Ch., \& Jones, G. (2001). Administración Estratégica, un Enfoque Integrado. Tercera Edición. Editorial Martha Suárez, Colombia.

Males, A. (2001). Proyecto de Turismo sustentable con comunidades Indígenas del Ecuador. MIT - OIT, Quito.

Ministerio de Turismo. (2002). Turismo y Desarrollo Sostenible, Quito, Ecuador.

Ministerio de Turismo. (2001). Ecuador Principales Indicadores Turísticos, Quito, Ecuador.

Municipio de Pastaza. (2005-2009). Plan de Desarrollo Turístico Local.

Rocher, G. (1980). Introducción a la Sociología General. Editorial Herder, Barcelona España.

Scholes, R.J., \& Biggs, R. (Eds.). (2004). Ecosystem Services in Southern Africa.

\section{Páginas de Internet}

http://www. wikipedia.org/wiki/Área protegida

http://www.monografias.com/trabajos11/bioltrece/bioltrece.shtml http://www.kuyima.com/seco/concepts.html

http:/www.sectur.pue.gob.mx/desarrollo/desarrolloculturaturisitca. htm Elementos de la Cultura Turística.

http://www.vivecuador.com/html2/esp/pastaza.es.him

http://www.pastaza.com

www.uta.edu.ec.

http://www.definicion.de conservación

http://nelasv.blogspot.com/

http://www.buenastareas.com/ensayos/que-es-la-conservacion-ambiental-y/1748173.html

http://es.wikipedia.org/wiki/Recurso_natural

http://www.definicionabc.com/geografia/recursos-naturales.php

http://www.peruecologico.com.pe/lib c15 t05.htm

$\mathrm{http}: / /$ sobreconceptos.com/selva

http://wikipedia.org/wiki/Selva

http://es.wikipedia.org/wiki/Reservas_ecologicas

http://es.wikipedia.org/wiki/Turismo

http://definicion.de/turismo/

http://www.todacultura.com/turismo/index.htm

http://ccqc.pangea.org/cast/sosteni/soscast.htm)

http://www.unep.org/gc/gc23/documents/Ecuador-desarrollo.pdf http://es.wikipedia.org/wiki/Jardín_botánico 